\title{
Solitary fibrous tumor: a clinicopathological study of 110 cases and proposed risk assessment model
}

\author{
Elizabeth G Demicco ${ }^{1}$, Min S Park ${ }^{2}$, Dejka M Araujo ${ }^{2}$, Patricia S Fox ${ }^{3}$, Roland L Bassett ${ }^{3}$, \\ Raphael E Pollock ${ }^{4}$, Alexander J Lazar ${ }^{1,5}$ and Wei-Lien Wang ${ }^{1}$ \\ ${ }^{1}$ Department of Pathology, The University of Texas M.D. Anderson Cancer Center, Houston, TX, USA; \\ ${ }^{2}$ Department of Sarcoma Medical Oncology, The University of Texas M.D. Anderson Cancer Center, Houston, \\ TX, USA; ${ }^{3}$ Department of Biostatistics, The University of Texas M.D. Anderson Cancer Center, Houston, TX, \\ USA; ${ }^{4}$ Department of Surgery, The University of Texas M.D. Anderson Cancer Center, Houston, Houston, TX, \\ USA and ${ }^{5}$ Department of Sarcoma Research Center, The University of Texas M.D. Anderson Cancer Center, \\ Houston, TX, USA
}

\begin{abstract}
Solitary fibrous tumor represents a spectrum of mesenchymal tumors, encompassing tumors previously termed hemangiopericytoma, which are classified as having intermediate biological potential (rarely metastasizing) in the 2002 World Health Organization classification scheme. Few series have reported on clinicopathological predictors with outcome data and formal statistical analysis in a large series of primary tumors as a single unified entity. Institutional pathology records were reviewed to identify primary solitary fibrous tumor cases, and histological sections and clinical records reviewed for canonical prognostic indicators, including patient age, tumor size, mitotic index, tumor cellularity, nuclear pleomorphism, and tumor necrosis. Patients $(n=103)$ with resected primary solitary fibrous tumor were identified (excluding meningeal tumors). The most common sites of occurrence were abdomen and pleura; these tumors were larger than those occurring in the extremities, head and neck or trunk, but did not demonstrate significant outcome differences. Overall 5- and 10-year metastasis-free rates were 74 and $55 \%$, respectively, while 5 - and 10 -year disease-specific survival rates were 89 and $73 \%$. Patient age, tumor size, and mitotic index predicted both time to metastasis and disease-specific mortality, while necrosis predicted metastasis only. A risk stratification model based on age, size, and mitotic index clearly delineated patients at high risk for poor outcomes. While small tumors with low mitotic rates are highly unlikely to metastasize, large tumors $\geq 15 \mathrm{~cm}$, which occur in patients $\geq 55$ years, with mitotic figures $\geq 4 / 10$ high-power fields require close follow-up and have a high risk of both metastasis and death. Modern Pathology (2012) 25, 1298-1306; doi:10.1038/modpathol.2012.83; published online 11 May 2012
\end{abstract}

Keywords: clinicopathological factors; hemangiopericytoma; metastasis; risk assessment; solitary fibrous tumor

Solitary fibrous tumor represents a single spectrum of mesenchymal tumors, of which hemangiopericytoma is a now considered a cellular phenotypic variant. These tumors, presumed to be of fibroblastic differentiation, most commonly affect adults and can occur at any site. ${ }^{1}$ Classically, solitary fibrous tumors are composed of variably pleomorphic spindle

Correspondence: Dr W-L Wang, MD, Department of Pathology, The University of Texas M.D. Anderson Cancer Center, 1515 Holcombe Blvd, Unit 085, Houston, TX 77030, USA.

E-mail: wlwang@mdanderson.org

Received 26 January 2012; revised 12 March 2012; accepted 13 March 2012; published online 11 May 2012 cells admixed with collagen, and arranged haphazardly ('patternless') or in short fascicles, while tumors within the previously separated hemangiopericytoma spectrum are composed of ovoid, monomorphic cells with thin-walled anastomosing vessels. Histological features of both can be seen in the same tumor, and both are CD34 reactive and have a dilated 'staghorn'-like vascular network, supporting the notion of a single tumor spectrum. ${ }^{1}$ Thus, once considered to be distinct entities, hemangiopericytoma is now considered to represent a cellular variant within the spectrum of solitary fibrous tumor. For the purposes of our study, the term hemangiopericytoma is only used in reference to historical 
studies or when discussing particular morphological features.

Solitary fibrous tumors are categorized as intermediate biological potential with a low risk of metastasis and relatively indolent course under the 2002 WHO Classification. ${ }^{1}$ However, the clinical behavior of individual tumors is notoriously difficult to predict. Furthermore, criteria for malignancy have not been consistent between published series, and the historical attempts at the division of hemangiopericytoma and solitary fibrous tumor has prevented investigations into this class of tumors as an unified spectrum or entity. ${ }^{2-8}$ Several histopathological criteria have been reported to be useful for determining malignancy in solitary fibrous tumor. These include increased tumor size, mitotic count, cellularity, presence of hemorrhage/necrosis, nuclear pleomorphism, ${ }^{2,3,9-11}$ and presence of sharply demarcated anaplastic/poorly differentiated foci (also regarded by some as 'dedifferentiation'). ${ }^{12}$ Few immunohistochemical and no molecular markers are known to have prognostic significance.

Few studies have examined the reported risk factors in a large series of primary solitary fibrous tumors, with formal, rigorous statistical analysis. ${ }^{10}$ Therefore, we evaluated both histopathological and clinicopathological factors in a large series of primary solitary fibrous tumors with clinical follow-up, including tumors previously classified as hemangiopericytoma, and devised a novel, clinically applicable risk stratification model for metastasis.

\section{Materials and methods}

\section{Patents and tumor tissues}

Upon Institutional Review Board review approval, 275 cases of primary non-meningeal solitary fibrous tumors, including cases previously diagnosed as hemangiopericytoma, were identified from the pathology files of The University of Texas M.D. Anderson Cancer Center from 1986-2009. Meningeal hemangiopericytomas, known to have a poor outcome relative to solitary fibrous tumor arising elsewhere in the body, ${ }^{13,14}$ were excluded. So-called 'sinonasal hemangiopericytoma' (aka. sinonasal glomangiopericytoma) were also excluded, although true solitary fibrous tumors arising in the sinus were included after histological review $(n=4)$. From the remaining cases, 110 cases of primary soft tissue $(n=79)$ and pleural solitary fibrous tumors $(n=31)$ were identified, which had both clinical follow-up and slides available for histological review.

\section{Evaluation of histopathological variables}

Available H\&E slides (between 1 and 27 slides/case) were confirmed by at least one experienced soft tissue pathologist. Tumors were scored for mitotic figures, cellularity, nuclear pleomorphism, and presence of necrosis (Figure 1). Mitotic index was calculated per 10 high-power fields $(\times 400)$, utilizing the highest count of 3-5 areas scored (depending on the number of available slides). The most cellular area of the tumor was scored for cellularity on a 3 -point scale from $1=$ low (tumor predominately composed of sclerotic collagen bands with scattered, compressed spindle cells) to $2=$ moderate (many areas of increased cellularity with cells adjacent to one another) to $3=$ high (hypercellular tumor, with areas of nuclear overlap). Pleomorphism was scored on a 3 -point scale from $1=$ low (cells monomorphic, with uniform nuclear features) to $2=$ moderate (increased nuclear pleomorphism, more prominent nucleoli, and rare multinucleated cells) to 3 (hyperchromatic nuclei present with foci of marked pleomorphism and bizarre cells). Areas of abrupt change from classic solitary fibrous tumor morphology to diffuse high-grade sarcoma were regarded as poorly differentiated (also referred to by some as 'dedifferentiation'). Necrosis/ hemorrhage was scored as minimal $(<10 \%)$ or positive $(\geq 10 \%)$, based on available histological sections.

\section{Clinical information}

Clinical information including patient demographics, tumor size, treatment, and patient outcome was retrieved from electronic patient medical records and tabulated for correlative analysis. Sites of primary tumors were categorized as pleural/intrathoracic, extremity, head and neck, intra-abdominal/pelvic, or superficial trunk. Local recurrence was defined as tumor involving the tumor bed or scar of previous surgery, while metastatic disease included both distant metastases to other organs as well as local sites, including peritoneal or pleural spread. Death from disease was defined as death from inexorable disease progression.

\section{Statistical analyses}

Total follow-up time and time to local recurrence, metastasis, and disease-specific death were calculated from the date of surgical resection. Patients with unknown recurrence or metastasis outcomes or cause of death were censored at date of last assessment for recurrence or metastasis and date of last contact, respectively. Kaplan-Meier analysis was used to estimate survival, and the log-rank test was used to assess differences by groups. Cox proportional hazards regression methodology was used to analyze the association between survival and histopathological and clinical factors. The Wilcoxon rank sum test and Fisher's exact test were used to assess association between continuous or 

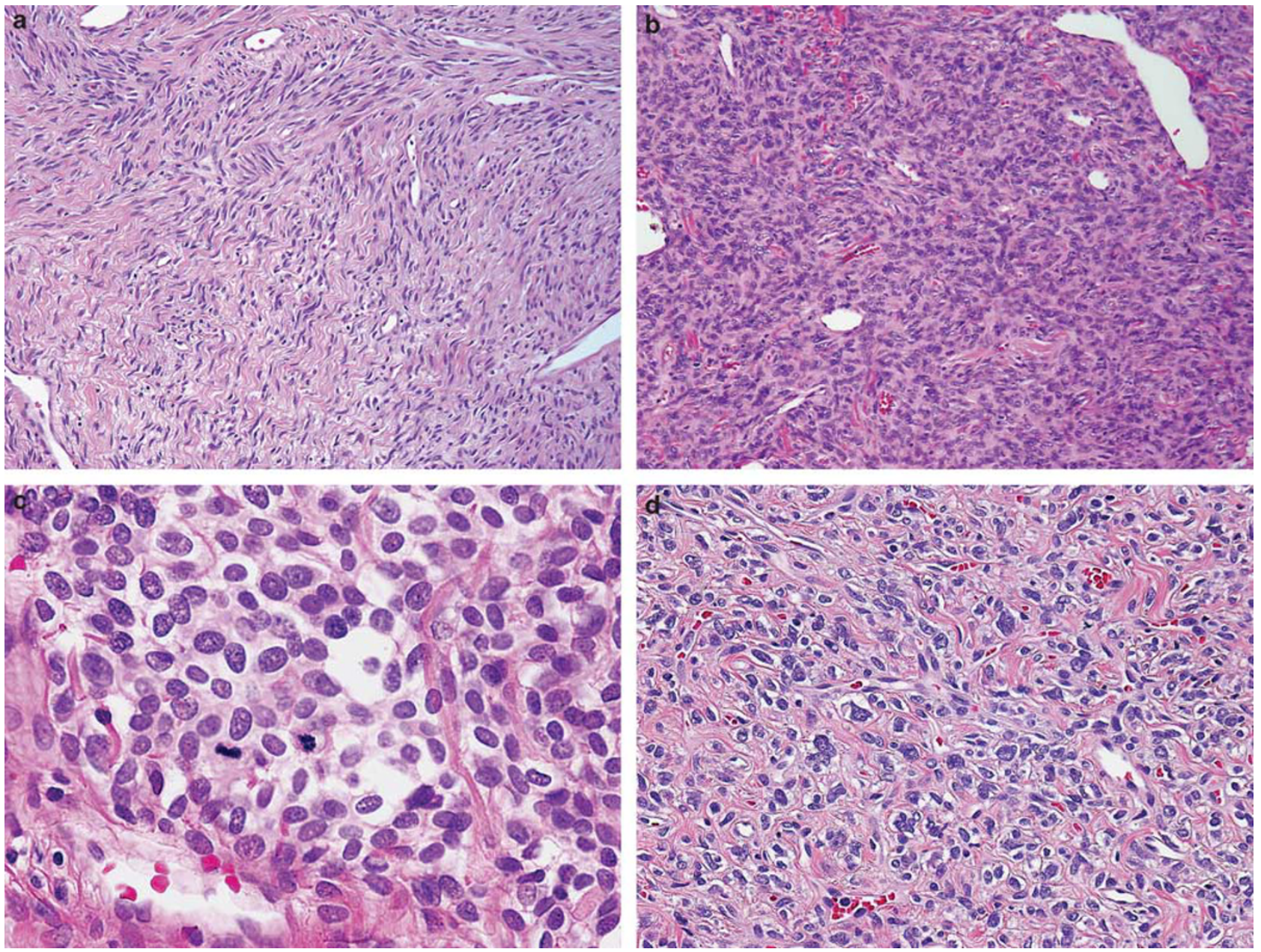

Figure 1 Histopathological parameters. (a) Low cellularity with prominent collagen bands $(\times 100)$. (b) High cellularity with overlapping nuclei and decreased collagen $(\times 100)$. (c) Mitotic activity $(\times 400)$. (d) Marked nuclear pleomorphism $(\times 200)$.

categorical variables and binary outcomes, respectively.

\section{Results}

\section{Clinical features}

The initial population included 110 patients, whose clinical features are detailed in Table 1. Primary tumor resection with curative intent was performed in 103 patients; these were used for outcome analysis, while the remaining 7 non-resected cases were excluded. Patients resected with curative intent included 52 women and 51 men, with a median age of 52 years (range, 19-81) at diagnosis. Diagnoses included 64 cases considered closer to the classical solitary fibrous tumor end of the spectrum, and 39 cases closer to classical hemangiopericytoma, although features of both could be seen in some cases. Tumors most frequently arose in the abdomen/pelvis (33 cases, 32\%), followed by pleura (29 cases, 28\%), extremity (17 cases, 16\%), head and neck and trunk (12 cases each, 12\%).

\section{Pathological features}

Tumor sizes ranged from 1 to $40 \mathrm{~cm}$ (median, 10.3) (Table 2). Abdominal and pleural tumors were significantly larger than those in other sites (median size 13 vs $6.8 \mathrm{~cm}, P<0.0001$ ). Pleural-based tumors presented about a decade later in life compared with all other sites $(P<0.0001)$.

Most tumors were at least moderately cellular, with only 3 cases demonstrating uniformly low cellularity and 51 cases being highly cellular. Necrosis was present in 37 cases, marked nuclear pleomorphism in 12, and distinct, sharply demarcated poorly differentiated areas were seen in 3 cases. Mitotic figures ranged from 0 to 23/10 high-power fields (median, 2).

\section{Treatment and outcome}

A total of 9 out of 103 patients underwent preoperative therapy with chemotherapy, radiotherapy, or combination chemotherapy and radiotherapy. An additional 7 patients underwent postoperative 
Table 1 Patient demographics

\begin{tabular}{|c|c|c|c|}
\hline & All patients $(\mathrm{n}=110)^{\mathrm{a}}$ & $\begin{array}{l}\text { Patients with resected } \\
\text { tumors }(\mathrm{n}=103)\end{array}$ & $\begin{array}{c}\text { Patients with unresectable disease } \\
\text { at diagnosis }(\mathrm{n}=5)\end{array}$ \\
\hline Age at diagnosis, years (median (range)) & $53(19-83)$ & $52(19-81)$ & $58(46-72)$ \\
\hline Gender (M:F) & $55: 55$ & $51: 52$ & $2: 3$ \\
\hline \multicolumn{4}{|l|}{ Ethnicity (n (\%)) } \\
\hline Caucasian & $82(75 \%)$ & $77(75 \%)$ & $4(80 \%)$ \\
\hline Hispanic & $12(11 \%)$ & $11(10 \%)$ & $1(20 \%)$ \\
\hline Asian & $7(6 \%)$ & 7 (7\%) & 0 \\
\hline African-American & $6(5 \%)$ & $5(5 \%)$ & 0 \\
\hline Unknown & $3(3 \%)$ & $3(3 \%)$ & 0 \\
\hline \multicolumn{4}{|l|}{ Site $(\mathrm{n}(\%))$} \\
\hline Intra-abdominal & $37(34 \%)$ & $33(32 \%)$ & $4(80 \%)$ \\
\hline Pleural & $31(28 \%)$ & $29(28 \%)$ & 0 \\
\hline Extremity & $18(16 \%)$ & $17(16 \%)$ & $1(20 \%)$ \\
\hline Head and neck & $12(11 \%)$ & $12(12 \%)$ & 0 \\
\hline Trunk & $12(11 \%)$ & $12(12 \%)$ & 0 \\
\hline
\end{tabular}

${ }^{\mathrm{a}}$ In addition to patients with resected tumors and those with unresectable disease, two patients with incidental small pleural solitary fibrous tumors discovered during work-up for other malignancies opted for follow-up rather than resection.

Table 2 Clinicopathological features of solitary fibrous tumor by site

\begin{tabular}{|c|c|c|c|c|c|c|}
\hline Feature & $\begin{array}{l}\text { All sites } \\
(\mathrm{n}=103)\end{array}$ & $\begin{array}{l}\text { Abdomen/pelvis } \\
(\mathrm{n}=33)\end{array}$ & $\begin{array}{l}\text { Pleura } \\
(\mathrm{n}=29)\end{array}$ & $\begin{array}{c}\text { Extremity } \\
(\mathrm{n}=17)\end{array}$ & $\begin{array}{c}\text { Trunk } \\
(\mathrm{n}=12)\end{array}$ & $\begin{array}{l}\text { Head/neck } \\
\quad(\mathrm{n}=12)\end{array}$ \\
\hline Male:female & $51: 52$ & $16: 17$ & $16: 13$ & $8: 9$ & $5: 7$ & $6: 6$ \\
\hline Age at diagnosis (years) & $53(19-83)$ & $51(23-75)$ & $61(32-81)$ & $53(31-69)$ & $48(19-78)$ & $41(28-69)$ \\
\hline Tumor size $(\mathrm{cm})$ & $10.3(1-40)$ & $12.5(2.5-40)$ & $15(1-30)$ & $8.7(2-18)$ & $7(1-10.5)$ & $4(1.5-7)$ \\
\hline Mitoses/10 HPF & $2(0-23)$ & $3(0-21)$ & $2(0-23)$ & $2(0-15)$ & $2(0-7)$ & $2(1-13)$ \\
\hline High cellularity & $51(50 \%)$ & $16(48 \%)$ & $14(48 \%)$ & $6(35 \%)$ & $7(58 \%)$ & $8(67 \%)$ \\
\hline High pleomorphism & $12(12 \%)$ & $5(15 \%)$ & $2(7 \%)$ & $1(6 \%)$ & $2(17 \%)$ & $2(17 \%)$ \\
\hline Necrosis & $37(36 \%)$ & $17(52 \%)$ & $12(41 \%)$ & $5(29 \%)$ & $3(25 \%)$ & $0(0 \%)$ \\
\hline
\end{tabular}

The numbers provided are median (range) or count (column percentage).

radiotherapy. Margin status was known in 61 cases, of which 47 were negative, 8 positive, and 6 close $(<0.1 \mathrm{~cm})$.

Median follow-up time after surgery was 48 months (range 1 day-279 months). Tumors reoccurred as local recurrence only $(n=4)$, local recurrence and metastasis $(n=6)$, and metastasis alone $(n=20)$ (Table 3). Overall 5- and 10-year metastasis-free rates were 74 and $55 \%$, respectively. Local, unilateral intrapleural or peritoneal metastases arose subsequently to eight pleural and three intra-abdominal primaries, respectively, while the remainder metastasized to distant organs. In 21 cases, metastases involved a single organ, although metastatic foci could be multiple. Five patients developed metastases to multiple organs. The lung/pleura was the most common site of metastasis, as a single site of metastasis in 16 cases or as one of the multiple sites in three cases. In patients with observed local recurrence or metastasis, median times to recurrence and metastasis were 84 months (range 12-195) and 36 months (range 4-228), respectively.

Over the course of follow-up, 21 patients died; 16 of these deaths occurred in patients with metastatic disease. In 5 patients without metastatic disease, death was due to complications of surgery after primary tumor resection $(n=1)$, unrelated causes $(n=3)$, and unknown causes $(n=1)$. In patients with metastatic disease, deaths were due to disease progression $(n=13)$, remote secondary complications of therapy $(n=2)$, and unknown causes $(n=1)$. Both overall survival and disease-specific survival were negatively associated with recurrent/metastatic disease $(P<0.0001)$.

\section{Clinicopathological features and outcome of non- resected tumors}

Five patients (median age 58, range 45-71) presented with unresectable disease at diagnoses due to metastasis $(n=4)$ or extensive local intra-abdominal disease $(n=1)$. Sites of primary disease in the four metastatic cases included abdomen $(n=3)$ and extremity $(n=1)$; primary tumor size ranged from 10-15.3 cm (median 13). All five patients died of disease at a median of 19 months (range 14-36), due to metastases to multiple sites, including the liver $(n=3)$ and lung $(n=2)$ or soft tissue $(n=1)$, lung metastases only, or local spread without metastasis 
Table 3 Outcomes by site

\begin{tabular}{|c|c|c|c|c|c|c|}
\hline Outcome & $\begin{array}{l}\text { All with outcomes } \\
\qquad(\mathrm{n}=101)\end{array}$ & $\begin{array}{l}\text { Abdomen/pelvis } \\
(\mathrm{n}=33)\end{array}$ & $\begin{array}{l}\text { Pleura } \\
(\mathrm{n}=29)\end{array}$ & $\begin{array}{l}\text { Extremity } \\
(\mathrm{n}=16)\end{array}$ & $\begin{array}{c}\text { Trunk } \\
(\mathrm{n}=11)\end{array}$ & $\begin{array}{l}\text { Head/neck } \\
(\mathrm{n}=12)\end{array}$ \\
\hline Local recurrence (n (\%)) & $10(10 \%)$ & $3(9 \%)$ & $3(10 \%)$ & $1(6 \%)$ & 0 & $3(25 \%)$ \\
\hline $\begin{array}{l}\text { Time to local recurrence } \\
\text { (months) }\end{array}$ & $84(12-195)$ & $21(12-102)$ & $141(15-195)$ & 89 & - & $79(35-192)$ \\
\hline Metastasis (n (\%)) & $26(26 \%)$ & $10(30 \%)$ & $9(31 \%)$ & $3(19 \%)$ & $2(18 \%)$ & $2(17 \%)$ \\
\hline $\begin{array}{l}\text { Time to first metastasis } \\
\text { (months) }^{\mathrm{a}}\end{array}$ & $36(4-228)$ & $45(6-102)$ & $30(4-119)$ & $36(10-162)$ & 8,15 & 81,228 \\
\hline \multicolumn{7}{|l|}{ Sites of metastasis ${ }^{\mathrm{b}}$} \\
\hline Lung/pleura & 19 & 4 & 9 & 3 & 1 & 2 \\
\hline Liver & 4 & 2 & & & 2 & \\
\hline Bone & 3 & 2 & & & 1 & \\
\hline Peritoneum & 3 & 3 & & & & \\
\hline Brain & 2 & 1 & & & 1 & \\
\hline Soft tissue & 1 & 1 & & & & \\
\hline Kidney & 1 & 1 & & & & \\
\hline
\end{tabular}

One patient died the day after surgical resection owing to complications of surgery.

${ }^{\mathrm{a}}$ Times to local recurrence or metastasis include patients with known recurrence/metastasis only. Median time (range) provided except in trunk and head/neck where only two patients developed metastases.

${ }^{\mathrm{b}}$ Sites of metastasis includes all sites of metastasis in patients with multiple metastases.

in the patient with unresectable local disease. An additional two patients were diagnosed with incidental $(3-4.2 \mathrm{~cm})$ pleural tumors during work-up for unrelated causes and opted for surveillance rather than resection; they remained alive without evidence of progression at 18 and 39 months, respectively.

\section{Characteristics of resected tumors with poor outcomes}

Primary tumors that went on to metastasize occurred in older patients (median 61 vs 51 years, $P=0.0043$ ), were larger (median 16.2 vs $8.7 \mathrm{~cm} ; P<0.0001$ ), had increased mitotic activity (median 6/10 high-power fields vs 2/10 high-power fields; $P<0.0001$ ), and were more likely to have necrosis $(11 / 26,58 \%$ vs 22 / $77,29 \% ; P=0.017$ ) (Figure 2).

Primary tumors in patients who died of disease also occurred in older patients (median 62 vs 51 years, $P=0.014$ ), showed increased size (median 23.5 vs $9 \mathrm{~cm}, P<0.0001$ ), and had higher mitotic activity (median 6/10 high-power fields vs 2/10 high-power fields, $P<0.0001)$. Necrosis was not significantly different $(6 / 13,46 \%$ vs 29/88, 33\% $P=0.53$ ).

Tumors previously diagnosed as hemangiopericytomas were equally likely to metastasize as those diagnosed as classic solitary fibrous tumors. There were no significant differences in time to metastasis or mortality based on the site of primary, overall cellularity or pleomorphism. All three cases with poorly differentiated foci (dedifferentiation) metastasized.

Among 61 patients with known margin status, positive or close margins were not significantly different between tumors that recurred locally or metastasized and those that did not. Local recurrence was more frequently seen in tumors that metastasized compared with those that did not (6/26, 23\% vs 4/75, 5\% $P=.017)$, and was identified before metastasis in four cases and at time of metastasis in two.

Factors predictive of time to metastasis included continuous age at diagnosis $(\mathrm{HR}=1.05 ; 95 \% \mathrm{CI}$ : 1.02-1.08; $P=0.0014)$, continuous tumor size ( $\mathrm{HR}=$ 1.09; 95\% CI: 1.05-1.13; $P<0.0001$ ), continuous mitotic count $(\mathrm{HR}=1.15 ; 95 \%$ CI: 1.07-1.22; $P<0.0001)$, and presence of necrosis $(\mathrm{HR}=3.22$; 95\% CI: 1.44-7.21; $P=0.0044)$. Binary cutoffs of age $\geq 55$ years, tumor size $\geq 15 \mathrm{~cm}$, and mitotic count $\geq 4$ for the multivariate model were chosen based on low $P$-values from univariate models and clinical significance (Table 4). Size $\geq 15$ and mitotic figures $\geq 4$ remained predictive in the multivariate Cox model (HR 7.31, 95\% CI: 2.55-20.98, $P=0.0002$ and HR 3.88, 95\% CI: $1.35-11.15, P=0.0119$, respectively).

Owing to the low number of metastases, multivariate analysis with more than two variables could not be performed.

\section{Risk stratification model}

A 3--tiered model stratifying our population by overall risk of metastasis (low, moderate, or high) was developed. Scores were assigned for age $(<55$ $v s \geq 55)$, tumor size $(<5,5$ to $<10,10$ to $<15$, or $\geq 15 \mathrm{~cm})$, and mitotic figures $(0,1-3$, or $\geq 4)$, and total scores tabulated to determine the risk of aggressive disease (Table 5). Necrosis was excluded from this model owing to the difficulty in reliably discerning in all cases tumor necrosis from necrosis secondary to neoadjuvant treatment or infarct-type necrosis. In 82 patients with data available for all 3 variables, risk was categorized as low $(n=28)$, moderate $(n=31)$, and high $(n=23)$. By log-rank analysis, there was a significant difference in both time to metastasis and time to disease-specific death 

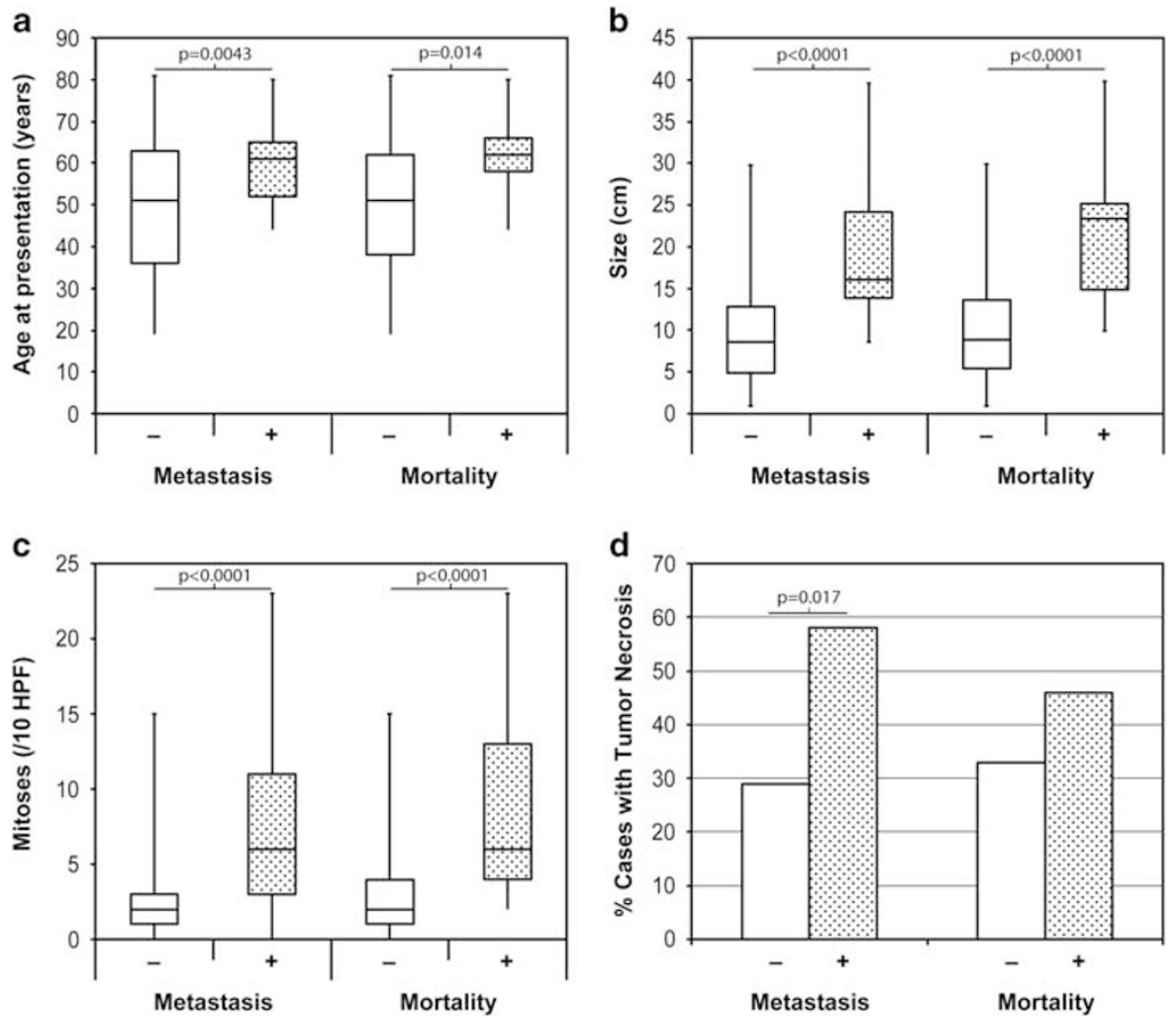

Figure 2 Clinicopathological variables associated with tumor metastasis and mortality. (a-c) Box and whisker plots. Boxes represent 25th-75th percentiles and horizontal line indicates the median. Whiskers indicate the highest and lowest outlier values. Patients who were event-free at last follow-up are indicated with $(-)$, while $(+)$ indicates patients with development of metastasis or disease-specific mortality. (a) Age. (b) Size. (c) Mitotic activity. (d) Percentage of cases with tumor necrosis.

Table 4 Univariate Cox model results for time to metastasis

\begin{tabular}{lccr}
\hline & $H R$ & $95 \% C I$ & \multicolumn{1}{c}{$\mathrm{P}$} \\
\hline Age $\geqslant 55$ years & 5.45 & $2.12-14.05$ & 0.0004 \\
Size $\geqslant 15 \mathrm{~cm}$ & 9.78 & $3.50-27.33$ & $<0.0001$ \\
Mitotic figures $\geqslant 4 / 10$ & 4.59 & $1.99-10.58$ & 0.0003 \\
high-power fields & & & \\
Necrosis & 3.22 & $1.44-7.21$ & 0.0044 \\
\hline
\end{tabular}

by risk group $(P<0.001)$ (Figure 3). No patients in the low-risk group developed metastasis or died of disease, over a median follow-up of 50 months (range 1-134). The 5-year metastasis-free rates in moderate and high-risk groups were 77 and $15 \%$, respectively, while 10--year metastasis-free rates were 64 and $0 \%$, over median follow-up times of 47 months (0-185) and 31 months (3-117), respectively. The 5-year disease-specific survival rates in moderate and high-risk groups were 93 and $60 \%$, respectively, while 10-year disease-specific survival rates were 93 and $0 \%$, respectively.

\section{Discussion}

To the best of our knowledge, this is one of the largest series to assess prognostic clinicopathologi-
Table 5 Risk stratification model

\begin{tabular}{lc}
\hline Risk factor & Score \\
\hline $\begin{array}{l}\text { Age } \\
\quad<55\end{array}$ & 0 \\
$\geqslant 55$ & 1 \\
& \\
Tumor size (cm) & 0 \\
$\quad<5$ & 1 \\
5 to $<10$ & 2 \\
10 to $<15$ & 3 \\
$\geqslant 15$ & \\
Mitotic figures (/10 high-power fields) & 0 \\
0 & 1 \\
$1-3$ & 2 \\
$\geqslant 4$ & Total score \\
Risk & $0-2$ \\
\hline Low & $3-4$ \\
Moderate & $5-6$ \\
\hline
\end{tabular}

cal predictors for the entire solitary fibrous tumor spectrum exclusively in primary tumors. Our 5- and 10-year disease-specific survivals were 89 and $73 \%$, respectively. Historically, hemangiopericytomas have been reported to have a worse prognosis than 

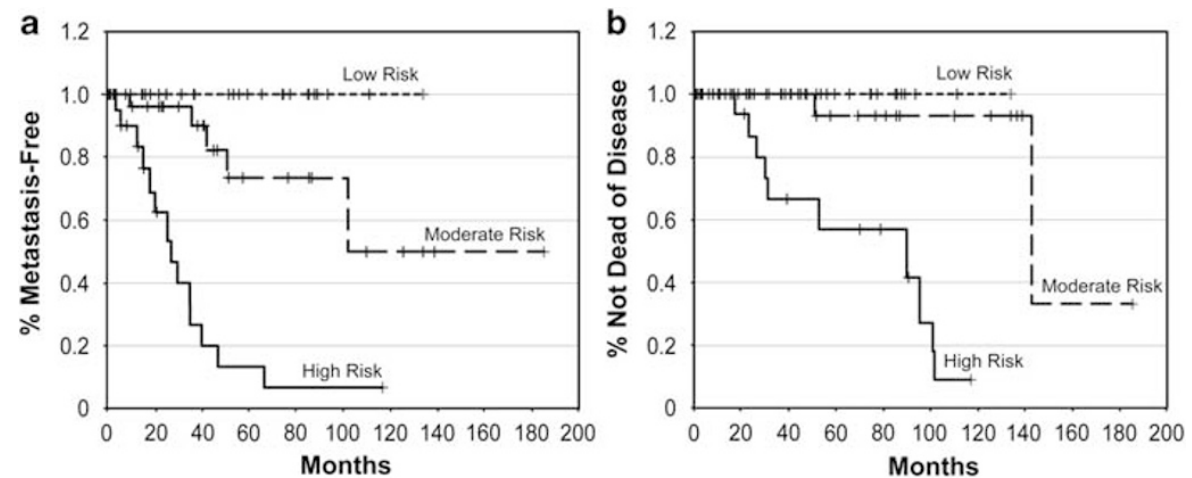

Figure 3 Risk stratification defines patients at risk for metastasis or death. (a) Kaplan-Meier plot for time to metastasis by risk group, $P<0.001$. (b) Kaplan-Meier plot for time to disease-specific mortality by risk group, $P<0.001$.

solitary fibrous tumor, with 5- and 10-year hemangiopericytoma survival rates as low as 59 and $47-$ $70 \%$, respectively, ${ }^{3,15}$ and up to $48 \%$ disease-specific mortality. ${ }^{16}$ In pleural solitary fibrous tumor, recurrence and mortality are reportedly predicted by both histology and resectability; typically, benign pedunculated tumors excised with clear margins have a recurrence rate of $2 \%$, while sessile malignant solitary fibrous tumors may recur up to $63 \%$ of the time, resulting in a $30 \%$ mortality rate. ${ }^{8,17}$ Extrathoracic solitary fibrous tumors behave similarly; diseasespecific death accounted for only 3 patients in one series of 75 patients ${ }^{10}$ and no patients in a series of 24 cases with up to 20 -year follow-up. ${ }^{18}$ In another large study of 92 extrathoracic solitary fibrous tumors, 5 of 10 cases with atypical features metastasized and an additional 3 tumors locally recurred; clinical follow-up was not provided for the remaining 82 tumors. ${ }^{11}$ Our findings of a poor 10-year disease-specific survival of $73 \%$ is likely due to referral bias at a major sarcoma treatment center with a population skewed toward more aggressive tumors. In fact, our rates are in keeping with those reported specifically for cases historically diagnosed as hemangiopericytoma and malignant solitary fibrous tumor. In our study, both cases previously considered to represent hemangiopericytoma and more classic solitary fibrous tumor had similar frequencies of poor outcomes, supporting the case to reclassify these tumors as a single entity.

In our series, 5- and 10-year rates of metastasis were 74 and $55 \%$, respectively, with metastasis occurring in $26 / 101(26 \%)$ patients. This rate was much higher than recently reported in one series of 79 cases of solitary fibrous tumor in which only 4 metastasized (5\%), ${ }^{10}$ and one of 39 pleural solitary fibrous tumor in which only 1 recurred. ${ }^{19}$ However, older series also found higher metastatic rates in hemangiopericytoma and pleural solitary fibrous tumor-from 18 to $50 \%{ }^{2,3,16,20}$ This variability may be due in part to differences in follow-up time as well as referral bias. Adequate follow-up time is required to determine metastatic frequency. McMaster et al found that $\sim 10 \%$ of metastases occurred after 5 event-free years, while Vallat-Decouvelaere et al found that $40 \%$ of metastases occurred after 5 years. ${ }^{11,16}$ Consequently, studies with short followup may not accurately reflect metastatic potential of tumors with malignant histological features, ${ }^{7}$ although even in studies with longer follow-up, tumors defined as malignant using historical criteria did not metastasize. ${ }^{19}$ In our study, $50 \%$ of metastases occurred after 36 months, but could present up to 19 years after initial resection.

Many of our patients underwent resection of the primary tumor at outside institutions, and we were unable to compile margin status in all cases. Gold et $a l^{10}$ found significant correlation between positive margins and eventual metastasis in soft tissue solitary fibrous tumor, while margin status is a well-described prognostic factor in pleural solitary fibrous tumor. ${ }^{8}$ This may have had a role in the high metastatic rate in our series as well. However, among the cases in which margin status was known, we did not find positive margins to be more frequent in tumors that recurred or metastasized.

We examined several previously reported risk factors. In our series, the strongest predictors of time to tumor metastasis and disease-specific death were patient age at presentation, tumor size, and mitotic index. Necrosis was also predictive of time to metastasis but not time to disease-specific death.

Patients in our series tended to have larger tumors $(>10 \mathrm{~cm})$ than those reported in the literature. ${ }^{3-7,10,18,20-22}$ This may be due to the large number of intra-abdominal tumors in our cohort. Previous studies have suggested that tumor size $>10 \mathrm{~cm}$ is predictive of poor prognosis. ${ }^{2,10}$ This also holds true in our cohort, although we found a cutoff size of $15 \mathrm{~cm}$ to be the most predictive of metastasis, likely owing to the large tumors in our study.

Mitotic activity is another reported risk factor for disease progression, with 4 mitotic figures/10 highpower fields being the historical cutoff in the literature for both hemangiopericytoma ${ }^{3}$ and pleural and soft tissue solitary fibrous tumor. ${ }^{2,6,10}$ Our data confirmed that any mitotic activity increased risk when examined as a continuous variable and that four mitotic figures/high-power fields is a strong categorical or discontinuous predictor of metastasis. 
Unlike several previous studies, we found no prognostic role for tumor cellularity or pleomorphism. One reason for this discrepancy may be due to the difficulty in establishing uniform histological parameters based on cellularity and pleomorphism applicable to both ends of the solitary fibrous tumor spectrum. In our study, moderate pleomorphism and cellularity were the norm in classic solitary fibrous tumors, while hemangiopericytomatous variants were more likely to have minimal pleomorphism, but high cellularity. Previous reports focus on cellularity in solitary fibrous tumor ${ }^{10}$ or pleomorphism in hemangiopericytoma ${ }^{16}$ together with mitotic activity as the criteria for malignancy. One instance in which cellularity and pleomorphism can be significant is in poorly differentiated areas (dedifferentiation), which occurs as an undifferentiated highgrade anaplastic sarcoma sharply demarcated from areas of conventional solitary fibrous tumor. Mosquera and Fletcher ${ }^{12}$ found that $3 / 7$ patients in their series died of disease and had a high risk of metastases and recurrence. All three patients with focal poorly differentiated areas in our study ultimately died after the anaplastic component metastasized. In this context, these factors portend a poor prognosis.

The anatomic site of primary tumors has also been reported to predict outcome. In our series, pleural tumors had a high rate of local (intrapleural and mediastinal) metastasis, but rarely metastasized out of the hemithorax. These findings are in keeping with previous studies of malignant pleural solitary fibrous tumors. ${ }^{2}$ In contrast, abdominal tumors behaved aggressively, with extraperitoneal metastases and metastases in multiple organs. The association of intra-abdominal hemangiopericytoma with poor outcomes has been described, ${ }^{5}$ and it has been suggested that all intra-abdominal hemangiopericytoma be treated as potentially malignant. ${ }^{5}$ Despite being larger, we found no statistically significant difference in time to metastasis between tumors arising in abdominal and pleural sites vs those that arose in other sites in our study. Our findings suggest that larger tumor size and mitotic activity may be more important than site, though this could be due to our limited sample sizes and lack of statistical power.

Using statistically significant and clinically important risk factors, we developed a risk stratification model for metastases, which divided our patient population roughly into thirds-a low-risk group in which no cases metastasized, a high-risk group in which nearly all cases metastasized, and an intermediate risk group likely comprised of a mixture of low- and high-risk tumors. Given that metastasis correlated with death from disease, this model was also used to identify patients at risk of disease-specific death.

Combining elements of grading (mitotic activity as in Fédération Nationale des Centres de Lutte Contre le Cancer $^{23}$ grading system) or staging systems (tumor size as in the American Joint Committee on Cancer $^{24}$ staging system) with clinical factors (patient age) is analogous to other risk assessment models such as gastrointestinal stromal tumor ${ }^{25}$ where mitotic count, tumor size, and anatomic site are used to determine the likelihood of aggressive disease. This is also similar to other prognostic nomograms for sarcoma, which combine mixtures of grading, staging, and clinical information, such as age and anatomic site. ${ }^{26}$ External validation is needed to fully test and refine our risk assessment model.

Other biomarkers have been previously explored for prognosis. However, little is still known about the genetic and molecular events responsible for the pathogenesis of solitary fibrous tumor and how these may relate to clinically determined risk factors. A few immunohistochemical or molecular biomarkers, including p53, telomerase activity, cyclin expression, and Ki67, have been suggested to have prognostic significance. ${ }^{27,28}$ Larger solitary fibrous tumors have been associated with more genomic copy number alterations than small tumors, suggesting a possible link to aggressive behavior. ${ }^{29}$ Future investigations to characterize potential biomarkers of solitary fibrous tumor are needed.

Our study was a retrospective study, and therefore subject to some limitations. As a tertiary referral center, many of our patients presented to our institution with advanced disease, for which primary tumors were then reviewed retrospectively. Thus, our study may overrepresent large and aggressive solitary fibrous tumors that required specialized management, as well as those with poor outcomes. As previously mentioned, margin status and size were not always available. Finally, while patients were treated similarly (surgery with curative intent), this was not done in a prospective and uniform setting and a few patients did also receive chemotherapy and/or radiation, though neither of these modalities has well-documented efficacy to prevent or manage metastatic disease. Despite these limitations, we were able to identify and confirm similar prognostic factors seen in previous studies.

In summary, we present a large series of primary solitary fibrous tumors with clinical follow-up and association of clinicopathological factors with metastasis and survival. Using these analyses, we created a risk stratification model which, with further validation, may prove to be useful in future evaluation of solitary fibrous tumors.

\section{Acknowledgements}

We would like to acknowledge $\mathrm{Kim} \mathrm{Vu}$ for her expert assistance in the figures. This work was supported in part by the Cancer Center Support Grant (NCI Grant P30 CA016672) (PSF, RLB), The University of Texas M.D. Anderson Cancer Center funds for research (W-LW, AJL), and the PhysicianScientist Program (AJL), and the WWWW Foundation (QuadW) (MSP). 


\section{Disclosure/conflict of interest}

The authors declare no conflict of interest.

\section{References}

1 Guillou L, Fletcher JA, Fletcher CDM, et al. Extrapleural solitary fibrous tumor and haemangiopericytoma. In: Fletcher CDM, Unni KK, Mertens F (eds) World Health Organization Classification of Tumours. Pathology and Genetics of Tumours of Soft Tissue and Bone. IARC Press: Lyon, 2002, pp 86-90.

2 England DM, Hochholzer L, McCarthy MJ. Localized benign and malignant fibrous tumors of the pleura. A clinicopathologic review of 223 cases. Am J Surg Pathol 1989;13:640-658.

3 Enzinger FM, Smith BH. Hemangiopericytoma. An analysis of 106 cases. Hum Pathol 1976;7:61-82.

4 Fisher JH. Hemangiopericytoma: a review of twenty cases. Can Med Assoc J 1960;83:1136-1139.

5 Goldman SM, Davidson AJ, Neal J. Retroperitoneal and pelvic hemangiopericytomas: clinical, radiologic, and pathologic correlation. Radiology 1988;168:13-17.

6 Insabato L, Siano M, Somma A, et al. Extrapleural solitary fibrous tumor: a clinicopathologic study of 19 cases. Int J Surg Pathol 2009;17:250-254.

7 Nielsen GP, O’Connell JX, Dickersin GR, et al. Solitary fibrous tumor of soft tissue: a report of 15 cases, including 5 malignant examples with light microscopic, immunohistochemical, and ultrastructural data. Mod Pathol 1997;10:1028-1037.

8 Robinson LA. Solitary fibrous tumor of the pleura. Cancer Control 2006;13:264-269.

9 Espat NJ, Lewis JJ, Leung D, et al. Conventional hemangiopericytoma: modern analysis of outcome. Cancer 2002;95:1746-1751.

10 Gold JS, Antonescu CR, Hajdu C, et al. Clinicopathologic correlates of solitary fibrous tumors. Cancer 2002;94:1057-1068.

11 Vallat-Decouvelaere AV, Dry SM, Fletcher CD. Atypical and malignant solitary fibrous tumors in extrathoracic locations: evidence of their comparability to intrathoracic tumors. Am J Surg Pathol 1998;22:1501-1511.

12 Mosquera JM, Fletcher CD. Expanding the spectrum of malignant progression in solitary fibrous tumors: a study of 8 cases with a discrete anaplastic component-is this dedifferentiated SFT? Am J Surg Pathol 2009;33:1314-1321.

13 Mena H, Ribas JL, Pezeshkpour GH, et al. Hemangiopericytoma of the central nervous system: a review of 94 cases. Hum Pathol 1991;22:84-91.

14 Goellner JR, Laws Jr ER, Soule EH, et al. Hemangiopericytoma of the meninges. Mayo Clinic experience. Am J Clin Pathol 1978;70:375-380.
15 Auguste LJ, Razack MS, Sako K. Hemangiopericytoma. J Surg Oncol 1982;20:260-264.

16 McMaster MJ, Soule EH, Ivins JC. Hemangiopericytoma. A clinicopathologic study and long-term follow up of 60 patients. Cancer 1975;36:2232-2244.

17 de Perrot M, Fischer S, Brundler MA, et al. Solitary fibrous tumors of the pleura. Ann Thorac Surg 2002; 74:285-293.

18 Hasegawa T, Matsuno Y, Shimoda T, et al. Extrathoracic solitary fibrous tumors: their histological variability and potentially aggressive behavior. Hum Pathol 1999;30:1464-1473.

19 Guo W, Xiao HL, Jiang YG, et al. Retrospective analysis for thirty-nine patients with solitary fibrous tumor of pleura and review of the literature. World J Surg Oncol 2011;9:134.

20 Briselli M, Mark EJ, Dickersin GR. Solitary fibrous tumors of the pleura: eight new cases and review of 360 cases in the literature. Cancer 1981;47:2678-2689.

21 Brunnemann RB, Ro JY, Ordonez NG, et al. Extrapleural solitary fibrous tumor: a clinicopathologic study of 24 cases. Mod Pathol 1999;12:1034-1042.

22 Cardillo G, Carbone L, Carleo F, et al. Solitary fibrous tumors of the pleura: an analysis of 110 patients treated in a single institution. Ann Thorac Surg 2009;88:1632-1637.

23 Trojani M, Contesso G, Coindre JM, et al. Soft-tissue sarcomas of adults; study of pathological prognostic variables and definition of a histopathological grading system. Int J Cancer 1984;33:37-42.

24 Edge SB, Byrd DR, Compton CC, et al. (eds) AJCC Cancer Staging Manual. Springer: New York, 2009.

25 Demetri GD, von Mehren M, Antonescu CR, et al. NCCN task force report: update on the management of patients with gastrointestinal stromal tumors. J Natl Compr Canc Netw 2010;8(Suppl 2):S1-S41; quiz S2-4.

26 Kattan MW, Leung DH, Brennan MF. Postoperative nomogram for 12-year sarcoma-specific death. J Clin Oncol 2002;20:791-796.

27 Schirosi L, Lantuejoul S, Cavazza A, et al. Pleuropulmonary solitary fibrous tumors: a clinicopathologic, immunohistochemical, and molecular study of 88 cases confirming the prognostic value of de Perrot staging system and p53 expression, and evaluating the role of c-kit, BRAF, PDGFRs (alpha/beta), c-met, and EGFR. Am J Surg Pathol 2008;32:1627-1642.

28 Miracco C, de Santi MM, Pacenti L, et al. Telomerase activity, Ki-67, cyclin D1 and A expression, and apoptosis in solitary fibrous tumors: additional features of a predictable course? Pathol Res Pract 2001;197:475-481.

29 Miettinen MM, el-Rifai W, Sarlomo-Rikala M, et al. Tumor size-related DNA copy number changes occur in solitary fibrous tumors but not in hemangiopericytomas. Mod Pathol 1997;10:1194-1200. 